heritage of humankind. Many countries have also subscribed to an International Undertaking on Plant Genetic Resources, formulated by FAO in 1983 to promote the free sharing of plant genetic resources. Such developments have resulted in a movement to promote "Farmers' Rights" and confer on farm families in the tropics and subtropics benefits similar to those given to plant breeders of developed nations under the PBR legislation. For it is largely farmers' efforts, in conscious and unconscious selection and maintenance of genetic diversity, that have led to the availability of such valuable genetic material to the plant breeders of developed countries. For the first time in agricultural history we are confronted with terms such as "Breeders' Rights" and "Farmers' Rights", as though the interests of breeders and farmers are somehow antagonistic.

In this context, Gene Banks and the World's Food is a timely publication. The authors are experienced scientists and have assembled a wide range of useful information and data covering all aspects of germplasm collection, characterization, conservation and use. They rightly stress that the availability of genetic variability by itself is no insurance against genetic vulnerability to pests and diseases, unless trained plant breeders use that variability effectively. Gene banks are of little value unless we know what genes they contain. The authors also refer to the new opportunities opened up by tissue culture and molecular biology for ex situ conservation, particularly of clonally propagated material. Genetic engineering techniques have enhanced the value of wild species and primitive cultivars, and DNA-transfer techniques are being rapidly refined.

The book will serve as an excellent source of information, particularly about the work being done by IARCs. The authors concentrate largely on the scientific aspects of conservation, and make only passing reference to matters relating to ownership, unrestricted access, and the long-term implications of plant patent rights in a two-page section titled "Seeds of Discord". A companion volume dealing with these items in an equally factual and lucid manner would be valuable. The subject of genetic resources is no longer the exclusive concern of scientists, as it was in 1932 when Vavilov first advocated plant-collecting expeditions.

M.S. Swaminathan is Director General of the International Rice Research Institute, $P O B O x$ 933, Manila. Philippines, and President of the International Union for the Conservation of Nature and Natural Resources.

\section{Summing it up}

\section{C.J.S. Clarke}

Encyclopedic Dictionary of Mathematics, 2nd Edn. The Mathematical Society of Japan, editor-in-chief Kiyosi Itô. MIT Press: 1987. Four volumes, pp.2,120. $\$ 350, £ 250$.

THE mathematical physicist is a jackdaw, constantly picking up bits of mathematical information; and for many of us the main source of such trinkets has long been the Encyclopedic Dictionary of Mathematics (EDM). Such tools of research are habitforming, and I for one would now be lost without the Dictionary. With the appearance of the second English edition (the original is in Japanese), any important part of main-stream mathematics, up to 1985-1986, can be located in a very comprehensive index, and pursued through an excellent system of crossreferences. In many cases the articles give all the information needed; in others, one learns enough to know where to go next and what to look for.

The new edition is a revision, rather than a rewriting, of the first, with expansion of the material by about half and reorganization of the topic-headings. A major improvement is the provision of references to English-language textbooks, in place of the original Japanese ones. The presentation has been made even clearer, particularly in the index. Much of the material, however, including the extensive tables in the appendices, has been reproduced unchanged (with some minor misprints preserved).

Many of the additions are predictable, and a comparison of the differences between the editions provides an interesting view of recent developments in mathematics. Differential topology was awarded only one page in the first edition; the topic now has nearly ten, and includes an account of the remarkable work of M.H. Freedman and S.K. Donaldson on probably be great in years to come. The article on the four-colour problem, which remains one of the few great mathematical problems that can be explained to a layman, has been rewritten, following its solution in 1976 by Haken, Appel and Koch. Some of the more fashionable aspects of mathematics are represented: fractals are there, with the definition of Hausdorff dimension (surprisingly omitted from the first edition), as are solitons, though not Bäcklund transformations. Renormalization group ideas enter with a new article, as does singularity theory, with accompanying tables. But one detects a certain puritanism in selection: the Monster group, though briefly

Away from the traditional centre of mathematics, and in applications, more 4-manifolds, whose impact on physics will mentioned, remains anonymous. controversial decisions had to be made as to what to include. One must applaud the addition of a new section (at the top level of classification) partly on computer science; but its scope is limited by also including combinatorics, coding theory and complexity theory, as well as mathematical models in biology. The treatment of all these topics is thin: one might have hoped for something on public key encryption and stochastic approaches to complexity theory (I could not find either), and more on Chaitin complexity and linguistic issues in computer science. But the section on automata, painfully inadequate in the first edition, has now been adequately rewritten.

There are sections on the history of mathematics (mainly organized bibliographically), on statistics and on operations research; and an extensive section of articles on mechanics and theoretical physics. This last includes a few pieces of a different character, sometimes nonmathematical, surveying an area of mathematical physics and aiming, one suspects, more at giving background information to the mathematician than detailed information for the physicist. These cover, for example, field theory, elementary particles and general relativity, and are well written within their limited scope.

Some of the more recent needs of the theoretical physicist have been met. The worker in string theory will find a modest enlargement of the material on Teichmueller spaces, which is now all collected in one article under that name. But a notable omission is super-mathematics: the graded versions of Lie algebras, Lie groups, manifolds and so on that have become so crucial for quantum gravity and string theory. The nearest one gets to these is a brief mention of Hopf coalgebras. Are the editors primly upset by appelation of 'super' to all these fields, or have they occult information that they will prove to be a passing craze?

A competitor to EDM is on the horizon, in the form of The Encyclopaedia of Mathematics, an updated and annotated translation of the Soviet Mathematical Encyclopaedia; the first volume has just been published by Reidel, and will be followed by another nine. As far as I can tell from the sample pages so far released (I have not seen the original), the coverage could be greater and the approach more careful pedagogically than that of EDM: the Soviet encyclopaedia explains, where EDM states. But for those who prefer a bird in the hand rather than waiting for the completion of publication of the Soviet work in 1990, the EDM is an essential buy for every library and for many private bookshelves.

C.J.S. Clarke is Professor of Applied Mathematics in the Faculty of Mathematical Studies, University of Southampton, Southampton SO9 $5 N H, U K$. 